[Vicino Oriente XXII (2018), pp. 121-140]

\title{
UNA BROCCHETTA EBURNEA DALLA NECROPOLI DI DOUÏMÈS: ARTIGIANATO FENICIO TRA NIMRUD E CARTAGINE
}

\author{
Michele Guirguis - Università di Sassari
}

Alla cara memoria di Paolo Bernardini, faro di illuminanti intuizioni

The review of an old discovery made by A.L. Delattre in the necropolis of Carthage (during 1896 excavations) offers the opportunity to reflect on a particular eburnean production (also documented in Nimrud in the Near East and Samos in Greece) that was inspired by a Red-Slip jug characteristic of the chronological horizons of the $9^{\text {th }}$ century BC Phoenicia, Levantine coast and Cyprus. This jug is inspired by metallic prototypes that were supposed to be luxury goods and objects of commerce, as in the case of (rare) ivory reproductions. The Carthaginian artefact could be an object of oriental production hoarded or acquired later in the West as an item of commercial exchange.

Keywords: Carthage; Nimrud; Phoenician; ivory; pottery

\section{IL REPERTO E IL CONTESTO DI RINVENIMENTO}

In questa nota intendiamo riproporre all'attenzione degli specialisti un singolare reperto rinvenuto dal R.P. Alfred Louis Delattre in un settore della necropoli arcaica di Cartagine alla fine del XIX secolo, ovvero una singolare brocchetta in avorio (fig. 1:A) ${ }^{1}$. L'interesse per questo reperto deriva, oltre che dal contesto di provenienza da una tomba cartaginese, principalmente dalla sua fisionomia, poiché risulta confrontabile con una particolare forma ceramica del Levante e, nello specifico, con una brocca di piccole dimensioni (fig. 1:B) documentata nell'areale della Fenicia centro-meridionale e di Cipro negli orizzonti cronologici del Ferro I, precisamente durante il IX secolo a.C.

Questo tipo di brocca è altresì ispirato a prototipi in metallo pregiato che dovevano rappresentare dei beni di lusso, oggetto di commercio e di forme di tesaurizzazione, come nel caso delle riproduzioni in avorio. I più diretti confronti per questa particolare realizzazione eburnea si rintracciano unicamente in due reperti provenienti, rispettivamente, da Nimrud, nel cuore dell'Assiria, e dall'Heraion di Samo, nella Grecia ionica. Anche i modelli della forma in avorio, riconoscibili in un particolare tipo ceramico a sua volta derivato dall'imitazione di esemplari metallici, appartengono ad un orizzonte cronologico compreso entro il IX secolo a.C. e ciò induce a riflettere sul contesto di rinvenimento cartaginese e sulle dinamiche di ricezione del reperto.

La piccola brocca eburnea proviene dal settore della necropoli cartaginese di Douïmès, scavato dal Delattre durante il biennio 1895-1896. Tra le trentatré tombe individuate nel mese di febbraio del 1896, il giorno 14 venne portata alla luce una semplice fossa terragna con un corredo composto da vasi greci (tra cui un alabastron figurato verosimilmente corinzio $)^{2}$ e alcuni scarabei: in pessimo stato di conservazione si rinvenne anche la

Delattre 1897, 128-129, fig. 83.

Dalla descrizione dell'oggetto si evince che l'alabastron «aux tons noir et violets est orné de deux cygnes becquetant l'extrémité pointue d'une palmette phénicienne»: Delattre 1897, 129; anche Philippe Berger, in relazione al settore di Douïmès indagato pochi anni prima, riferisce del rinvenimento di ceramiche corinzie e 
brocchetta in avorio oggetto di questo studio (fig. 2). Com'è noto, il settore di Douïmès assieme a quelli di Junon, della Byrsa e di Dermech - ha restituito alcuni lembi dell'ampia necropoli arcaica di Cartagine ${ }^{3}$ che tuttavia non consentono di risalire cronologicamente oltre la fine dell'VIII-metà del VII secolo a.C., mentre i settori più antichi finora individuati e databili al pieno VIII secolo a.C. si riferiscono ai resti di incinerazioni localizzate nel settore di Bir Messaouda ${ }^{4}$ e ad una tomba costruita nella Rue Astarté, risalendo il pendio nel medesimo versante sud-orientale di Byrsa ${ }^{5}$.

Grazie alle informazioni ricavabili dalla pubblicazione del 1897, la zona indagata dal Delattre al principio del 1896 restituì altre sepolture caratterizzate da corredi con presenza di oggetti in avorio che, in alcuni casi, trovano puntuali confronti nella documentazione orientale, di stile egittizzante e non; molti di questi oggetti sembrerebbero sensibilmente più antichi rispetto al contesto cronologico complessivo di VII-VI secolo a.C. delle sepolture cartaginesi, al pari della nostra brocchetta.

L’oggetto della presente nota è attualmente documentabile grazie all'illustrazione riportata nella pubblicazione originale del Delattre, successivamente riproposta da William Culican ${ }^{6}$ (figg. 1:A, 2:B), nonché attraverso una piccola immagine (fig. 2:A) collegata alla relativa scheda all'interno di un recente lavoro d'insieme sugli avori conservati nel Musée Nationale de Carthage, da cui si evince che attualmente la brocchetta è residua unicamente della vasca e del piede, avendo perso la porzione superiore del collo ${ }^{7}$. Rinvenuta in uno stato di conservazione precario, aveva originariamente un'altezza di circa $15,5 \mathrm{~cm}(10,5 \mathrm{~cm}$ residui) ed era caratterizzata dalle proporzioni dimensionali slanciate, che si sviluppano con un largo piede distinto, una pancia piriforme rovesciata, uno stretto collo tubolare con orlo trilobato e labbro sagomato con arrotondamento delle estremità; alcuni frammenti si riferiscono a sottili anse a doppio bastoncello innestate tra l'orlo e la spalla della vasca. Si segnala infine la sottigliezza delle pareti che, a dispetto delle dimensioni quasi miniaturistiche del reperto, suggeriscono una destinazione funzionale come contenitore di materie pregiate (profumi, balsami o liquidi).

Il colore chiaro e biancastro della superficie, a fronte di altri numerosi avori rinvenuti nei corredi delle tombe di Cartagine che appaiono colorati intenzionalmente in rosso oppure anneriti e alterati da processi chimico-fisici posteriori o da interventi intenzionali (cremazione, contatto con metalli, politura, erosione etc. $)^{8}$, è verosimilmente dovuto all'utilizzo di una materia prima ricavata da un giovane elefante, come recentemente ipotizzato $^{9}$.

di «ivory pieces artistically worked»: Berger 1898, 605; su queste sepolture scavate del Delattre vd. anche Gsell 1896, 449-452; 1898, 80-81; 1899, 40-41.

3 Benichou-Safar 1982; Chelbi 1985.

4 Chelbi - Maraoui Telmini - Docter 2006; Docter et al. 2006, 43-45, fig. 11; Maraoui Telmini - Chelbi - Docter 2013, 911-914.

Maraoui Telmini 2017.

Culican 1968, 281, fig. 2.

Khelifi 2017, 34 (n. 52, n. inv. 03-02-21-18)

Sottili lamine auree e pigmenti superficiali sono stati riconosciuti e analizzati su un'ampia serie di avori di età neo-assira conservati al Musée du Louvre: Reiche et al. 2013; Albéric et al. 2015

$9 \quad$ Khelifi 2017, 143 


\section{DA NIMRUD A SAMO: I CONFRONTI IN AVORIO E I PARALLELI TIPOLOGICI IN CERAMICA E} METALLO

\subsection{Il modello ceramico}

La fisionomia della brocca eburnea è caratterizzata da alcune particolarità che di seguito si segnalano: ampio piede distinto ad anello, lievemente concavo; vasca piriforme rovesciata, con spalla notevolmente arrotondata; stretto collo cilindrico tendente alla svasatura; bocca trilobata; sottili anse a doppio cannello impostate tra la spalla e l'orlo. La forma ricorda in maniera precisa le caratteristiche brocchette orientali del tipo "trefoilrimmed jug/decanter/pithcer" documentate nei corredi di alcune tombe della Fenicia centro-meridionale e soprattutto nell'areale di Tiro, nonché a Cipro, (fig. 3). Come vedremo, queste forme sembrerebbero ispirarsi a modelli in metallo, considerando la presenza di appendici plastiche che ricordano le analoghe realizzazioni della metallotecnica orientale $^{10}$.

Da Cipro provengono diversi esemplari probabilmente importati, rivestiti in Red-Slip e caratterizzati da un'attenta brunitura delle superfici; i migliori confronti sono documentati nella necropoli di Amatunte (fig. 3:H) e a Kourion (attribuzione incerta: fig. 3:G), entro corredi tombali inquadrati nel Cipro-Geometrico III e nelle fasi iniziale e media del Salamis horizons ${ }^{11}$. Nelle coste del Levante si segnalano le forme intere individuate nelle sepolture più arcaiche di Khaldé a sud di Beirut (fig. 3:C) ${ }^{12}$, nonché a Khirbet Silm (fig. 3:D) ${ }^{13}$ e a Joya (fig. 3:B $)^{14}$ nell'areale interno di Tiro. La stessa necropoli di Tiro (Al Bass) ha restituito due brocche (U51-3 e U61-5) individuate durante gli scavi del 1997 e quelli del 2002 (fig. 3:A) ${ }^{15}$ : entrambe le forme appartengono al Period II, collocabile cronologicamente entro l'ultimo quarto del IX secolo a.C. Le due deposizioni tirie si riferivano a tombe particolari nel panorama generale della necropoli: nel caso della Urn 51 veniva segnalata una «unusual furniture» ${ }^{16}$, mentre per la Urn 61 , sovrastata da una piccola stele lapidea con simbolo ankh, è stato rimarcato che «the size and wealth of the grave goods suggest that the buried was a personage of high status» ${ }^{17}$.

Questa particolare forma ceramica, studiata tra gli altri da M. Prausnitz e W. Culican ${ }^{18}$, è pienamente identificabile nella Form 300 di Lehmann ${ }^{19}$, nel tipo Jug $7^{20}$ e $\mathrm{Jv} 1 / \mathrm{Jv} 2 \mathrm{~d} 2$

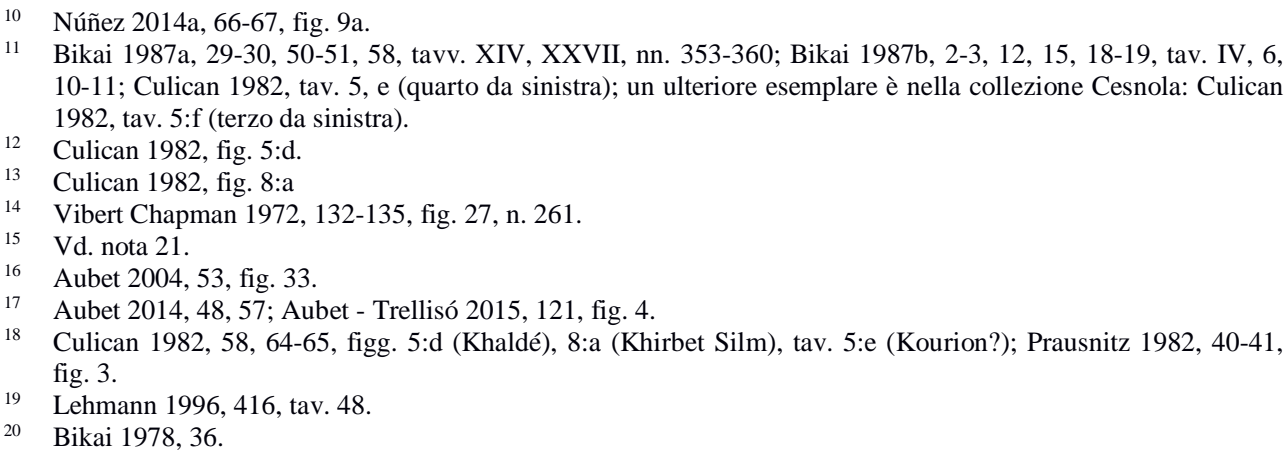


della necropoli di Tiro Al Bass ${ }^{21}$ e nel tipo JG1 I documentato anche nella necropoli di Akhziv (fig. 3:E) ${ }^{22}$ oltre che nel centro di Tell es-Safi/Gath (fig. 3:F) ${ }^{23}$. Tutti gli esemplari individuati mostrano delle proporzioni dimensionali bilanciate e si attestano su un'altezza tendenzialmente superiore ai $22 \mathrm{~cm}$, con alcuni esemplari di poco inferiori ai $30 \mathrm{~cm}$.

\subsection{I prototipi in metallo}

Come accennato sopra, sia le forme in ceramica che le riproduzioni di avorio, dovevano a loro volta ispirarsi ad esemplari metallici che, in realtà, risultano decisamente sottorappresentati, a differenze di altre forme contemporanee ${ }^{24}$ e delle più tarde realizzazioni dell'VIII-VII secolo a.C. che riproducevano le coeve brocche bilobate ${ }^{25}$. Tra le numerose forme chiuse in metallo, distribuite nell'areale vasto del Mediterraneo orientale, si segnalano pochi paralleli convincenti. Nella collezione Cesnola (da Kourion) è presente un esemplare in bronzo (fig. 4:A) particolarmente slanciato ${ }^{26}$, che tuttavia mostra uno stadio evolutivo della forma molto prossimo alle brocche bilobate in Red-Slip dell'VIII secolo a.C. Altrettanto può dirsi per un esemplare rinvenuto nella Idaean Cave sull'isola di Creta (fig. $4: B)^{27}$.

In ambito cartaginese, oltre alle produzioni cd. "rodie” (probabilmente locali) e alle più tarde realizzazioni di età punica ed ellenistica, si conosce una brocca in bronzo (fig. 4:C), sempre proveniente dagli scavi del Delattre nel medesimo settore di Douïmès, che tuttavia riproduce le fattezze di una brocca bilobata dell'inoltrato VI secolo a.C. richiamante, come già evidenziato da Javier Jiménez Ávila, le tipiche forme biconiche sarde del Sulcis durante il periodo tardo-arcaico ${ }^{28}$.

Tra i pochi confronti proponibili, secondo un'intuizione di Fulvia Lo Schiavo, può essere individuato un esemplare miniaturistico in bronzo proveniente dalla Sardegna nordoccidentale, rinvenuto nella cosiddetta "Aula rettangolare" del santuario di Monte S. Antonio a Siligo (SS) ${ }^{29}$. La brocchetta enea richiama tutte le caratteristiche delle coeve produzioni orientali in ceramica, ma se ne discosta per la presenza del fondo cuspidato (fig. 4:D-E). Anche l'inquadramento cronologico del reperto sardo riconduce ad un orizzonte che non oltrepassa il Bronzo Finale/Primo Ferro, collocabile dopo la seconda metà del X ed entro il IX secolo a.C. Inoltre la piccola oinochoe in bronzo è chiaramente una forma di provenienza esterna che non trova paralleli nella pur abbondante produzione nuragica del periodo, del resto ampiamente studiata proprio in relazione ai contatti con la metallurgia

21 Núñez 2004, 116, 316, fig. 173; Núñez 2008, 39-41, fig. 14; come specificato dallo stesso studioso (Núñez 2014b, 278, 348, 354, 369, nota 30), la brocca prima inquadrata nel tipo Jv1 risulterebbe ora inquadrabile nel tipo Jv 2d; da ultimo, per il corredo ceramico della U61: Núñez 2018, 11, fig. $5 a 5$.

22 Dayagi-Mendels 2002, 122; Mazar 2001, 26-27, fig. 9, 1.

23 Amiran 1969, 272, fig. 281, tav. 92; dal medesimo centro provengono altre produzioni ceramiche del tardo IX secolo a.C., di tradizione e - probabilmente - produzione fenicia, che mostrano di derivare la propria fisionomia da esemplari metallici, come nel caso della forma aperta edita in Maeir - Shai 2007.

24 Artzy 2006, tavv. 7-11.

25 Botto 2014; per ulteriori brocche metalliche di età arcaica, classica ed ellenistica: Morpurgo 2008; Picard 1995; Taloni 2015.

26 Matthäus 1985, n. 539; vd. anche Grau-Zimmermann 1978, n. K3.

27 Kourou 2012, 41, 49, fig. 1a.

28 Jiménez Ávila 2002, 63-64, figg. 38-39; Botto 2014, 398.

29 Lo Schiavo 2000, 219-221, fig. 6:4 a-b; vd. anche Sanna - Lo Schiavo 1992, 197-203, fig. 52:a. 
cipriota. Nell'ambito di una generale riconsiderazione sulle sequenze dei ripostigli sardi, Nicola Ialongo conferma l'inquadramento della brocchetta enea, definita come un “pendaglio miniaturistico” (tipo 191; MSA-137), nel Primo Ferro 2A (locale Fase 7) ${ }^{30}$

\subsection{I confronti in avorio}

Come già notato in alcuni lavori precedenti di Maria Taloni ${ }^{31}$, i diretti confronti per il reperto cartaginese sono rintracciabili in analoghe realizzazioni in avorio provenienti da Nimrud e dall'Heraion di Samo. Nel contesto insulare greco si conosce un esemplare, frammentario, caratterizzato dall'attacco inferiore dell'ansa configurato a palmetta (fig. 5:A), rinvenuto nel deposito del pozzo $G$ del santuario assieme ad altri avori e bronzi figurati di importazione orientale, tra i quali si può almeno segnalare la presenza di oggetti d'uso (pettine) e di una protome maschile con barba appuntita e tripartita (fig. 5:B), secondo un modello di ascendenza orientale che si riflette, altresì, in una serie di bronzi antropomorfi di fattura levantina ${ }^{32}$, nell'ambito di un contesto santuariale che recepisce anche alcuni calderoni bronzei con protomi di grifoni di probabile produzione urartea ${ }^{33}$. Ma il rinvenimento dal tempio di Hera senza dubbio più importante ai fini dell'inquadramento dei rapporti internazionali che stanno alla base della ricezione di questi oggetti, è il significativo elemento di bardatura equina (frontalino) con iscrizione aramaica di Hazael di Damasco (fig. 5:C) ${ }^{34}$, sovrano di Aram nella seconda metà del IX sec. a.C. ${ }^{35}$

La brocchetta di Nimrud (fig. 6:A), appartenente al gruppo di avori individuato da William Kennet Loftus (1821-1858) durante gli scavi del 1854-1855 rimasti pressoché inediti $^{36}$ (da cui lo studio di R.D. Barnett del $1957^{37}$ ), è un documento prezioso per ragionare sugli aspetti cronologici e sui rapporti tra Cartagine e l'area levantina in relazione alla ricezione di prodotti raffinati dell'artigianato. Il recipiente in avorio, frammentario, si caratterizza per il particolare attacco inferiore dell'ansa configurato a forma di palmetta come nell'esemplare da Samo, mentre le peculiarità morfologiche si richiamano alle forme ceramiche discusse nel paragrafo precedente.

30 Essa proviene dalla US19 dell'edificio 9 in cui vennero rinvenute alcune ceramiche di produzione fenicia, anche con rivestimenti in Red-Slip: Ialongo 2010, 367, figg. 25:6-8 (vol. I); 190, fig. 24 (vol. II).

31 Taloni 2012, 80-81, fig. 2, e-g; Taloni 2013, 310-312, fig. 1, 2-3; Taloni 2015 123-124, 128, 130, figg. 4 , 11:3; si deve tuttavia segnalare che la brocchetta assira viene erroneamente attribuita ai depositi del SW Palace, con una cronologia di fine VIII secolo a.C.; cfr. anche Freyer-Schauenburg 1966, 95-97, tav. 27:b; Botto 2014, 401, nota 40.

32 Freyer-Schauenburg 1966, 95-97, tav. 27:a (brocchetta); tav. 17 (protome maschile barbuta); vd. anche Jiménez Ávila 2015, 205-206, fig. 8:2.

33 Kyrieleis 1990, 19-21, figg. 4-5; Lipiński 2004, 156-158.

34 Amadasi Guzzo 1987; Fales 2006; Naso 2015, 375.

35 Un oggetto simile (paraocchi) con iscrizione menzionante il sovrano siriano proviene anche dal tempio di Apollo ad Eretria, a ulteriore dimostrazione dei contatti privilegiati tra l'Eubea e le coste siro-levantine, di cui un chiaro riflesso è desumibile anche sotto il profilo dei primi grammata documentati, come nel caso della nota iscrizione semitica KPLŠ; il contesto di rinvenimento, non ricostruibile con certezza, dovrebbe riferirsi ad un deposito del tardo VII secolo a.C. con oggetti appartenenti originariamente ai primi contesti abitativi delle élites eretriesi di fine IX-inizi VIII secolo a.C.: Boffa 2013, 32-34, fig. 4; vd. anche Mazarakis Ainian 1997, 59-62.

36 Mallowan 1952, 3, 15-19; Mallowan 1954, 71; Barnett 1935, 186-188; Herrmann - Laidlaw 2013a, 85-86.

37 Barnett 1957; Mallowan 1966; Oates - Oates 2004, 6, 227-228. 
Il reperto venne rinvenuto nel palazzo South-East ("Burnt Palace”) di Nimrud che, analogamente ai più noti palazzi NW, SW e ad altri complessi di edifici dell'acropoli, subì duramente le conseguenze del dirompente incendio occorso durante i convulsi anni del tracollo dell'Impero assiro, precisamente durante il sacco della città (avvenuto più o meno contemporaneamente a quello di Ninive nel 612 a.C.). La colorazione scura del reperto si deve pertanto all'azione del fuoco che ha conferito questo aspetto alla pressoché totalità degli avori rinvenuti dal Loftus a Nimrud ${ }^{38}$. Dal punto di vista cronologico, le costruzioni dei complessi che insistono nel settore SE dell'acropoli (fig. 6:B) e nello specifico il primo palazzo neo-assiro ${ }^{39}$ (Fasi D-F; 1A), successivamente denominato Burnt Palace, devono essere attribuiti ad Aššurnașirpal II (ca. 885-859 a.C.) ${ }^{40}$ o, meno probabilmente, al figlio Shalmaneser III (ca. 858-824 a.C.).

Come segnalava R.D. Barnett, gli avori del cosiddetto "Loftus group" apparterrebbero dunque alla prima metà del IX secolo a.C. e sarebbero coerenti con la prima fase monumentale del complesso: «all were made (...) in the early ninth century, and installed in a small treasure chamber in a building of Ashurnasirpal during his lifetime, and remained there untouched by his obedient successors until the destruction of the empire itself» ${ }^{41}$. Sebbene l'incendio sia ovviamente attribuibile all'ultima fase del Palazzo, anche Irene J. Winter ipotizza che gli avori possano essere «considerably earlier and kept as heirlooms», proponendo dei confronti con le iconografie documentate nell'alta Siria (Tell Halaf) ${ }^{42}$.

Gli avori levantini - al fianco di quelli prodotti localmente e al netto di quelli realizzati da artigiani "deportati” - giungevano nella capitale assira, almeno a partire dal IX secolo a.C., attraverso differenti modalità di acquisizione e come beni di lusso che venivano commercializzati ma anche, com'è noto, saccheggiati e requisiti come forme di tributo soprattutto nel caso del mobilio intarsiato, non di rado realizzato con legno di cedro e/o ebano. Nel caso della brocchetta in avorio del "Loftus group" potrebbe trattarsi di un contenitore funzionale, facente parte degli arredi del Palazzo e utilizzato durante i complessi cerimoniali di corte.

In maniera suggestiva, si possono qui richiamare diverse placchette in avorio, sempre da Nimrud, che restituiscono un'iconografia particolare e diverse varianti di un tema narrativo legato alla libagione e ai rituali che prevedevano l'uso di una brocchetta, che appare rappresentata in forma slanciata, con sottili anse e ampia bocca lobata: i pannelli con "Pharaohs with sceptres and jugs" provengono soprattutto dalla Room SW.11/12 del Forte Salmanassar (fig. 7:A) ${ }^{43}$, ma diversi paralleli sono rintracciabili anche nei materiali da SW.37 (fig. 7:C-F) ${ }^{44}$ e da SW.7 (fig. 7:B) ${ }^{45}$; i confronti per questo genere di raffigurazioni,

38 Sempre a Nimrud, dalla stanza T10 del Fort Shalmaneser proviene «a large number of heavily burnt ivories and the long bones of an elephant»: Curtis 2008, 57; in generale le tracce degli incendi e i depositi di cenere sono molto diffusi in tutti gli edifici dell'acropoli.

39 Fasi D-F in Oates - Reid 1956; Fasi 1A-2A in Mallowan 1954; il rifacimento successivo (Fase F) è attribuibile a Adad-nirari III (ca. 823-811 a.C.): Oates - Oates 2004, 124-130; Pedde 2012, 856; Reade 2011, 116.

40 Mallowan 1954, 63-65, pl. XI.

41 Barnett 1935, 198; sul diario di scavo del Loftus: Reade 2008, 5.

42 Winter 1976a, 15, nota 67; sull'argomento anche Barag 1983; Di Paolo 2009, 136-139.

43 Winter 1976b, 39-40, fig. 15 (ND. 11035); vd. anche Mallowan 1966, 506, 547-548, fig. 481.

44 Mallowan 1966, 542-544; pl. 4, 23-24; Herrmann - Laidlaw 2013b, 34-35, 57-58, fig. 2g.

45 Mallowan - Herrmann 1974, 10, 106, tav. XCV, 87: «youth wearing the triple atef crown» (Metropolitan Museum di New York, ND. 7579); Mallowan 1966, 506, fig. 412. 
di forte influenza levantina, possono essere riconosciuti in alcuni rilievi da Arslan Tash, Zinçirli, Karkemish, suggerendo l'appartenenza, in alcuni casi, allo stile "North Syrian” o "Syrian-Intermediate" in altri al "Classic Phoenician" ${ }^{46}$. Eccezionalmente, il medesimo tema figurativo con la presenza di una brocchetta è riprodotto sui due lati del noto naiskos proveniente da Sidone, databile tra 850-675 a.C., attualmente conservato nel Musée du Louvre (fig. 8:A) ${ }^{47}$, mentre in Occidente si ritrova su un rasoio da Cartagine (fig. 8:B) ${ }^{48}$, secondo un fenomeno di "transizione" figurativa già messa in evidenza per un altro tema iconografico documentato negli avori di Nimrud e riprodotto nei rasoi bronzei punici ${ }^{49}$. In relazione alla forma ceramica di riferimento, analoghe conclusioni sull'identificazione proposta in questa sede sono state recentemente avanzate da Eric Gubel: «the piriform juglets held by acolytes on another series of ivory panels illustrate a type already present amongst Phoenician imports at Tell es-Safi in the 9th century BCE, as well in the Salamis and Amathous horizons» ${ }^{50}$. Benché le figurazioni proposte possano solo ipoteticamente ricondurre alle reali forme vascolari effettivamente in uso tra IX e VIII secolo a.C., il tentativo di identificazione tra iconografie dei manufatti (talvolta molto precise) e oggetti concretamente esistiti può comunque condurre ad interessanti letture che sembrano mostrare una standardizzazione delle forme e delle modalità di rappresentazione di queste, specialmente nelle scene legate al consumo cerimoniale di vino durante i banchetti funerari ${ }^{51}$.

Un'ultima considerazione merita la particolare conformazione dell'attacco inferiore dell'ansa, riproducente una "palmetta" sia nella brocchetta eburnea di Samo che in quella di Nimrud: anche in questo caso, i confronti tipologici e la finezza dell'intaglio richiamano identiche configurazioni apprezzabili su un'ingente quantità di pannelli eburnei e anche su oggetti funzionali, come nell'esempio fornito da due frontlets in avorio provenienti dal pozzo AJ del Palazzo NW di Nimrud (fig. 9:A-B) e da un analogo oggetto in bronzo da Enkomi (fig. 9:C) ${ }^{52}$, nei quali la conformazione della palmetta è molto simile a quella intagliata nella brocchetta in avorio del Burnt Palace. Inoltre, come segnalato sopra, in forma isolata e funzionale le stesse palmette caratterizzeranno gli attacchi inferiori delle brocche fenicie e cipriote in argento e in bronzo (vd. supra).

\section{LONG-DISTANCE TRADE, COMMERCIO DI ANTIQUARIA O TESAURIZZAZIONE?}

Come appare evidente dai confronti proposti, la brocchetta in avorio da Cartagine imita quasi fedelmente, seppure in dimensioni inferiori, un particolare tipo di brocca trilobata in Red-Slip che non appare documentata oltre il IX secolo a.C. e che sembra costituire, al pari delle neck-ridge jug rispetto alle brocche con orlo espanso, il predecessore diretto delle brocche bilobate dell’VIII secolo a.C.

\footnotetext{
Herrmann - Laidlaw 2013b, 57-58, 89-90, fig. 4:j.

47 Aimé-Giron 1934; da ultimo: Gubel 2016, 174, fig. 9; vd. anche Herrmann - Laidlaw - Coffey 2009, 79-80, fig. 26.

48 Acquaro 1971, fig. 26, tav. XII, Ca 61; Cecchini 2005, 259-260, fig. 8.

49 Secci 2011.

50 Gubel 2000, 200-201, fig. 21:a-c.

51 Núñez 2018, 17-19, fig. 6; vd. anche Stampolidis 2001, 69

52 Herrmann - Laidlaw 2013a, 93-94, fig. 10; Gubel 2005, 123-133, figg. 13-14, 20.
} 
Il settore della necropoli di Douïmès da cui proviene il reperto in avorio si configura come uno dei più arcaici finora noti, anche se le descrizioni fornite dal suo scavatore consentono di circoscrivere la cronologia di numerose tombe tra la fine del VII e la prima metà del VI secolo a.C. Per tali motivi riteniamo comunque possibile che l'oggetto in avorio possa essere sensibilmente più antico del relativo contesto funerario e abbia dunque subito una forma di tesaurizzazione. Del resto anche a Cartagine non è insolito, proprio in relazione agli oggetti di produzione vicino-orientale o egizia, assistere a fenomeni di acquisizione e riutilizzo che appaiono molto evidenti soprattutto per altre categorie di manufatti, come gli scarabei, i sigilli cilindrici o le coppe metalliche, seppure se non mancano interessanti casi di manufatti eburnei di chiara ascendenza levantina e anche "mesopotamica" ${ }^{53}$. Secondo un'altra ipotesi il pezzo potrebbe fra parte di un circuito mercantile di antiquaria, tendente a veicolare verso Occidente oggetti di pregio più antichi anche di diversi secoli, secondo un fenomeno non insolito soprattutto nella prima metà del I millennio a.C.

Quanto alla localizzazione della bottega responsabile della realizzazione del pezzo, crediamo che l'abilità tecnica e i confronti stilistici indirizzino verso una manifattura orientale, probabilmente fenicia o più latamente del Levante meridionale. Il quesito sul centro di fabbricazione delle brocche si collega alla più generale tematica degli avori orientali e delle diverse correnti artistiche documentate soprattutto nei pannelli che decoravano gli arredi interni dei principali edifici assiri. Nel nostro caso, rimarcando l'ambientazione cronologica del tipo entro il IX secolo a.C., crediamo che potesse trattarsi di un'elaborazione di un centro fenicio; la lunga e complessa storia degli studi sulle produzioni eburnee del Vicino Oriente del I millennio a.C. ${ }^{54}$, consente infatti oggi di riconoscere, a lato di una serie di scuole e botteghe attive in diversi centri siro-palestinesi fin dall'età del Bronzo, anche dei centri di produzione localizzabili nell'areale della Fenicia e nel sud del Levante (e.g. Samaria), da cui il reperto di Nimrud venne verosimilmente saccheggiato per confluire nel nuovo palazzo del sovrano assiro Aššurnașirpal II.

Dopo le prime incursioni di Tiglath-pileser I nelle coste del "mare superiore" con i doni imprecisati ricevuti dalle città di Arado, Biblo e Sidone attorno al 1100 a.C., fu proprio sotto Aššurnașirpal II che vennero ottenuti i primi ingenti tributi, tra i quali è forse possibile riconoscere dell'avorio, anche se G. Bunnens ha espresso delle pesanti riserve sull'interpretazione del testo di riferimento, riconducendo il termine con significato di "denti/avorio" all'animale marino citato nel testo degli annali (nāhiru) piuttosto che ai denti di elefante (šinni pīri). Inoltre, sebbene la riscossione dei tributi dai "re della costa" continuò con il successore Shalmaneser III ( $18^{\circ}$ campagna dell’841 e durante il $21^{\circ}$ anno di regno nell'838 a.C.) e con i sovrani successivi, per la prima menzione certa dell'avorio come tributo proveniente dalla Fenicia occorre discendere cronologicamente fino ad Esarhaddon ${ }^{55}$.

In relazione ai contesti greci che hanno restituito avori e oggetti pregiati in metallo di provenienza orientale, Edward Lipiński sostiene che «these objects did not reach Samos

53 Si veda, ad esempio, il frammento in avorio decorato, non più documentabile perché disperso, studiato in Redissi 2007.

54 Cecchini - Mazzoni - Scigliuzzo 2009

55 Bunnens 1985, 126-128; sulla questione si veda anche: Fales 2017, 223-224, 250-255, nota 170. 
and Euboea directly from their first owner, in the second part of the $9^{\text {th }}$ century B.C., but were offered later to the temples as valuable objects acquired somehow by trade with SyroPhoenicia» ${ }^{56}$.

Considerando la non diffusa ricezione di oggetti pregiati di derivazione orientale nei contesti delle tombe cartaginesi (ricordiamo, ad esempio, una serie di cylinder seals neoassiri e una patera in bronzo), pensiamo che una forma di commercio secondario, con oggetti raffinati appartenenti a varie epoche e distribuiti in Occidente, possa aver condotto la brocchetta in avorio entro il corredo della sepoltura cartaginese, anche se rimane sullo sfondo l'alternativa ipotesi che la forma di tesaurizzazione sia da ricercarsi all'interno della stessa comunità cartaginese, con la presenza di un "cimelio di famiglia" che viene deposto dopo diverse generazioni come parte di un corredo funerario.

\section{BIBLIOGRAFIA}

ACQUARO, E.

$1971 \quad$ I rasoi punici (Studi Semitici 41), Roma 1971.

Alberic, M. - Müller, K. - Pichon, L. - Lemasson, Q. - Moignard, B. - Pacheco, C. - Fontan, E. REICHE, I.

2015 Non-invasive quantitative micro-PIXE-RBS/EBS/EBS imaging reveals the lost polychromy and gilding of the Neo-Assyrian ivories from the Louvre collection: Talanta 137 (2015), pp. 100-108.

AIME-GIRON, N.

1935 Un naos phénicien de Sidon: Bulletin de l'Institut Français d'Archéologie Orientale 34 (1935), pp. 31-42.

AMADASi GuZzo, M.G.

1987 Iscrizioni semitiche di nord-ovest in contesti greci e italici (X-VII sec. a.C.): Dialoghi di Archeologia 5, 2 (1987), pp. 13-27.

AMIRAN, R.

1969 Ancient Pottery of the Holy Land. From its beginnings in the Neolithic period to the end ARTZY, M. of the Iron Age, Jerusalem 1969.

2006 The Jatt Metal Hoard in Northern Canaanite/Phoenician and Cypriote Context (Cuadernos de Arqueología Mediterránea 14), Barcelona 2006.

AuBET, M.E.

2004 The Iron Age Cemetery: M.E. Aubet (ed.), The Phoenician Cemetery of Tyre-Al Bass. Excavations 1997-1999 (Bullettin d'Archéologie et d'Architecture Libanaise. Hors-Série 1), Beyrouth 2004, pp. 9-62.

2014 The Excavations 2002-2005: M.E. AuBET - F.J. NúÑEZ - L. Trellisó (eds.), The Phoenician Cemetery of Tyre-Al Bass II. Archaeological Seasons 2002-2005 (Bulletin d'Archéologie et d'Architecture Libanaise. Hors-Série 9), vol. I, Beyrouth 2014, pp. 7-54 Aubet, M.E. - TRELLisó, L.

2015 Pratiques funéraires à l’âge du Fer II au Liban: la nécropole de Tyr al-Bass: G. GeRNEZ (ed.), Funerary Customs in Lebanon from Prehistory to the Roman Period (Archaeology \& History in the Lebanon 40-41), Beyrouth 2015, pp. 118-134.

\footnotetext{
56 Lipiński 2004, 156.
} 
BARAG, D.

1983 Glass Inlays and the Classification and Dating of Ivories from the Ninth-Eight Centuries BARNETT, R.D. B.C.: Anatolian Studies 33 (1983), pp. 163-167.

1935 The Nimrud Ivories and the Art of the Phoenicians: Iraq 2, 2 (1935), pp. 179-210.

1957 Catalogue of the Nimrud Ivories in the British Museum, London 1957.

BENICHOU-SAFAR, H.

1982 Les tombes puniques de Carthage. Topographie, structures, inscriptions et rites funéraires, Paris 1982.

BERGER, P.

1898 The Excavations at Carthage: Annual Report of the Board of Regents of the Smithsonian Institution, june 30, 1898, pp. 601-614.

BIKAI, P.M.

1978 The Pottery of Tyre, Warminster 1978.

1987a The Phoenician Pottery of Cyprus (A.G. Leventis Foundation), Nicosia 1987.

1987b The Phoenician Pottery: V. KARAGEORGHIS - O. PICARD - C. TYTGAT (eds.), La nécropole d'Amathonte. Tombes 113-367 II. Céramiques non Chypriotes (Études Chypriotes VIII), Nicosie 1987, pp. 1-19.

BOFFA, G.

2013 Il santuario di Apollo ad Eretria. Osservazioni sulla documentazione epigrafica di età geometrica: L. GiARDino - G. TAgLiAmonte (edd.), Archeologia dei luoghi e delle pratiche di culto, Atti del Convegno (Cavallino, 26-27 gennaio 2012) (Bibliotheca Archaeologica 32), Bari 2013, pp. 31-43.

BONATZ, D.

2014 Art: H. NieHR (ed.), The Aramaeans in Ancient Syria (Handbook of Oriental Studies 106), Leiden - Boston 2014, pp. 205-253.

BotTo, $\mathrm{M}$

2014 Le oinochoai di tipo "fenicio-cipriota". Considerazioni sulla diffusione di una forma vascolare fra Oriente e Occidente mediterraneo: A. Lemaire (ed.), Phéniciens d'Orient et d'Occident. Mélanges Josette Elayi (Cahiers de l'Institut du Proche-Orient Ancien du Collège de France 2), Paris 2014, pp. 393-418.

BUNNENS, G.

1985 Le luxe phénicien d'après les inscriptions royales assyriennes: E. GUBEL - E. LIPINSKI (eds.), Studia Phoenicia III. Phoenicia and its Neighbours. Proceedings of the Colloquium held on the $9^{\text {th }}$ and $10^{\text {th }}$ of December 1983 at "Vrije Universiteit Brussel", Leuven 1985, pp. 121-133.

CECCHINI, S.M.

2005 The "suivant du char royal": A case of interaction between various genres of minor art: C.E. Suter - C. Uehlinger (eds.), Crafts and Images in Contact. Studies on Eastern Mediterranean art of the first millennium BCE (Orbis Biblicus et Orientalis 210), Göttingen 2005, pp. 243-264.

Cecchini, S.M. - Mazzoni, S. - Scigliuzzo, E.

2009 Syrian and Phoenician Ivories of the Early First Millennium BCE: Chronology, Regional Styles and Iconographic Repertoires, Patterns of Inter-regional Distribution. Acts of the International Workshop, Pisa, December $9^{\text {th }}-11^{\text {th }}$ 2004, Pisa 2009.

CHELBI, F

1985 Découverte d'un tombeau archaïque à Junon (Carthage): Reppal 1 (1985), pp. 95-119. 
Chelbi, F. - Maraoui Telmini, B. - Docter, R.F.

2006 Découverte d'une nécropole du VIIIe siècle av. J.-C. à Carthage: Bir Massouda. Rapport préliminaire sur les fouilles de l’Institut National du Patrimoine (Tunis) et l’Université de Gand: Cedac Carthage 22 (2006), pp. 13-15.

Culican, W.

1968 Quelques aperçus sur les ateliers phéniciens: Syria 45, 3-4 (1968), pp. 275-293.

1982 The Repertoire of Phoenician Pottery: H.G. NiEMEYeR (ed.), Phönizier im Western (Madrider Beiträge 8), Mainz 1982, pp. 45-82.

CURTIS, J.

2008 The British Museum Excavations at Nimrud in 1989: J.E. CurTIS - H. MCCALL - D. Collon - L. AL-Gailani WerR (eds.), New Light on Nimrud. Proceedings of the Nimrud DAYAGI-MENDELS, $\mathrm{M}$.

2002 The Akhziv Cemeteries. The Ben-Dor Excavations, 1941-1944 (Israel Antiquities Authority Reports 15), Jerusalem 2002.

DelattRe, A.L.

1897 La nécropole punique de Doümès (à Carthage). Fouilles de 1895 et 1896 (Mémoires de la Société Nationale des Antiquaires de France 1, LVI), Paris 1897.

Di PAOLO, $\mathrm{S}$.

2009 What production model can be deduced for first-millennium BC Syro-Phoenician ivories? Some observations, with analogies drawn from byzantine, early medieval and gothic material: S.M. CECCHINI - S. MAzzoni - E. Scigliuzzo, Syrian and Phoenician Ivories of the Early First Millennium BCE: Chronology, Regional Styles and Iconographic Repertoires, Patterns of Inter-regional Distribution. Acts of the International Workshop, Pisa, December $9^{\text {th }}-11^{\text {th }} 2004$, Pisa 2009, pp. 133-154.

Docter, R.F. - Chelbi, F. - Telmini, B.M. - Bechtold, B. - Ben Romdhane, H. - DeclercQ, V. - De Schacht, T. - Deweirdt, E. - De Wulf, A. - Fersi, L. - Frey-Kupper, S. - Garsallah, S. - Joosten, I. - Koens, H. - Mabrouk, J. - Redissi, T. - Chebbi, S.R. - Ryckbosch, K. - Schmidt, K. TAVERniers, B. - VAN KeRCKHOVE, J. - VERdONCK, L.

2006 Carthage Bir Massouda. Second preliminary report on the bilateral excavations of Ghent University and the Institut National du Patrimoine (2003-2004): BABesch 81 (2006), pp. 37-89.

FALES, M.F.

2006 Rivisitando l'iscrizione aramaica dell'Heraion di Samo: A. NASo (ed.), Stranieri e non cittadini nei santuari greci. Atti del Convegno Internazionale, Firenze 2006, pp. 230-252.

2017 Phoenicia in the Neo-Assyrian period. An updated overview: State Archives of Assyria Bullettin 23 (2017), pp. 181-295.

FREYER-SCHAUENBURG, B.

1966 Elfenbeine aus dem samischen Heraion, Hamburg 1966.

GABELLONE, F. - SCARDOZZI, G.

2007 Integrated technologies for the reconstructive study of Mesopotamian cultural heritage: The cases of Ur, Uruk and Nimrud: Archäologie und Computer 2006. Workshop 11, Wien 2007, pp. 1-46.

GRAU-ZiMMERMANN, B.

1978 Phönizische Metallkannen in den Orientalisiereden Horizonten des Mittelmeerraumes:

GSELL, S. Madrider Mitteilungen 19 (1978), pp. 161-218.

1896 Chronique archéologique africaine: Mélanges d'archéologie et d'histoire 16 (1896), pp. 441-490. 
1898 Chronique archéologique africaine: Mélanges d'archéologie et d'histoire 18 (1898), pp. 69-140.

1899 Chronique archéologique africaine: Mélanges d'archéologie et d'histoire 19 (1899), pp. 35-83.

GUBEL, E.

2000 Multicultural and multimedial aspects of early Phoenician art, c. 1200-675 BCE: C. UEHLINGER (ed.), Images as media. Sources for the cultural history of the Near East and the Eastern Mediterranean (Ist millennium BCE) (Orbis Biblicus et Orientalis 175), Göttingen 2000, pp. 185-214.

2005 Phoenician and Aramean bridle-harness decoration: Examples of cultural contact and innovation in the Eastern Mediterranean: C.E. SuTER - C. UeHLINGER (eds.), Crafts and Images in Contact. Studies on Eastern Mediterranean art of the first millennium BCE (Orbis Biblicus et Orientalis 210), Göttingen 2005, pp. 111-147.

2016 Crossing Continents: Phoenician Art and How to Read It: J. ARUZ - M. SEYmour (eds.), Assyria to Iberia. Art and Culture in The Iron Age (The Metropolitan Museum of Art Symposia), London 2016, pp. 168-179.

HERRMANN, G.

$1986 \quad$ Ivories from room SW 37 Fort Shalmaneser. Plates (Ivories from Nimrud [1949-1963] IV, 2), London 1986.

HERRMANN, G. - LAIDLAW S.

2013a Assyrian Nimrud and the Phoenicians: Archaeology International 16 (2013), pp. 84-95.

2013b Ivories from Rooms SW11/12 and T10 Fort Shalmaneser. Commentary and Catalogue (Ivories from Nimrud [1946-1963] VII, 1), London 2013.

Herrmann, G. - LaidLaW, S. - COFFEy, H.

2009 Ivories from The North West Palace (1845-1992) (Ivories from Nimrud VI), London 2009.

IALONGO, N.

2010 Il santuario nuragico di Monte S. Antonio di Siligo (SS). Studio analitico dei complessi cultuali della Sardegna protostorica, vol. I-II, Tesi di Dottorato Università "La Sapienza” di Roma, XXII Ciclo, Roma 2010.

JiMÉNEZ ÁVILA, J.

2002 La toréutica orientalizante en la Península Ibérica (Bibliotheca Archaeologica Hispana 16. Studia Hispano-Phoenicia 2), Madrid 2002.

2015 Figuras fenicias del Mediterráneo. Caracterización y novedades: J. JimÉNEz ÁviLA (ed.), Phoenician Bronzes in Mediterranean (Bibliotheca Archaeologica Hispana 45), Madrid 2015, pp. 197-230.

KHELIFI, L.

2017 Les ivoires à Carthage à l'époque phénico-punique, Tunis 2017.

KOUROU, N.

2012 Phoenicia, Cyprus and the Aegean in the Early Iron Age: J.N. Coldstream’s contribution and the current state of research: M. IAcovou (ed.), Cyprus and the Aegean in the Early Iron Age. The Legacy of Nicolas Coldstream, Nicosia 2012, pp. 33-51.

KYRIELEIS, $\mathrm{H}$.

1990 Samos and Some Aspects of Archaic Greek Bronze Casting: C. HuDson (ed.), Small Bronze Sculpture from the Ancient World, Papers Delivered at a Symposium Organized by the Departments of Antiquities and Antiquities Conservation and Held at the J. Paul Getty Museum, March 16-19, 1989, Malibu 1990, pp. 15-30. 
LEHMANN, G.

1996 Untersuchungen zur späten Eisenzeit in Syrien und Libanon. Stratigraphie und Keramikformen zwischen ca. 720 bis 300 v.Chr. (Altertumskunde des Vorderen Orients 5), Münster 1996.

LIPIŃSKI, E.

2004 Studia Phoenicia XVIII. Itineraria Phoenicia (Orientalia Lovaniensia Analecta 127), Leuven 2004

Lo SCHIAVO, F.

2000 Forme di contenitori di bronzo e di ceramica: documenti ed ipotesi: P. BARTOLONI - L. Campanella (eds.), La ceramica fenicia di Sardegna. Dati, problematiche, confronti. Atti del Primo Congresso Internazionale Sulcitano, Sant'Antioco 19-21 settembre 1997 (Collezione di Studi Fenici 40), Roma 2000, pp. 207-223.

MAEIR, A.M. - SHAI, I.

2007 An Iron Age IIA Phoenician-Style (?) fluted bowl from Tell es-Safi/Gath: a ceramic imitation of a metal prototype: Journal of the Serbian Archaeological Society 23 (2007), pp. 219-226.

MALLOWAN, M.E.L.

1952 The Excavations at Nimrud (Kalhu), 1951: Iraq 14, 1 (1952), pp. 1-23.

1954 The Excavations at Nimrud (Kalhu), 1953: Iraq 16, 1 (1954), pp. 59-114.

$1966 \quad$ Nimrud and its Remains, vol. I-II, London 1966.

MALlOWAN, M.E.L. - HERRMANN, G.

$1974 \quad$ Furniture from SW.7 Fort Shalmaneser. Commentary, Catalogue and Plates (Ivories from Nimrud [1949-1963] III), London 1974.

MARAOUI TELMini, B.

2017 Découverte d'une tombe construite du 8ème siècle av. J.-C., sur le versant sud-est de la colline de Byrsa à Carthage: Carthage Studies 8 (2017), pp. 47-72.

Maraoui Telmini, B. - Chelbi, F. - Docter, R.F.

2013 Les fouilles tuniso-belges du terrain Bir Massouda (2002-2005): contribution à la connaissance de la topographie de Carthage à l'époque archaïque: A.M. ARRUDA (ed.), Fenícios e púnicos, por terra e mar. Actas do VI Congresso Internacional de Estudos Fenícios e Púnicos (Estudos e memórias 6), vol. 2, Lisboa 2013, pp. 906-916.

MATTHÄUS, $\mathrm{H}$

1985 Metallgefässe und Gefässuntersätze det Bronzezeit, der Geometrischen und Archaïsschen Periode auf Cypern (Prähistorische Bronzefunde II, 8), Munich 1985.

MAZAR, E.

2001 The Phoenicians in Achziv. The Sothern Cemetery. Jerome L. Joss Expedition Final Report of the Excavations 1988-1990 (Cuadernos de Arqueología Mediterránea 7), Barcelona 2001.

MAZARAKIS AINIAN, A.

1997 From Rulers' Dwellings to Temples. Architecture, Religion and Society in Early Iron Age, Thessaloniki 1997.

MORPURGO, G.

2008 Note sulla produzione delle oinochoai bronzee con appliques configurate in ambito

NASO, A. punico: Byrsa 5, 1-2 (2008), pp. 129-154.

2015 Bronzi fenici e bronzi etruschi: J. JimÉNEz ÁvilA (ed.), Phoenician Bronzes in Mediterranean (Bibliotheca Archaeologica Hispana 45), Madrid 2015, pp. 375-393. 
NÚÑEZ, F.J.

2004 Catalogue of urns: M.E. AuBET (ed.), The Phoenician Cemetery of Tyre-Al Bass. Excavations 1997-1999 (Bullettin d'Archéologie et d'Architecture Libanaise, Hors-Série 1), Beyrouth 2004, pp. 63-206.

2014a Tyrian potters and their products: standardization and variation in the pottery of the al Bass cemetery: A. Kotsonas (ed.), Understanding Standardization and Variation in Mediterranean Ceramics. Mid $2^{\text {nd }}$ to late $1^{\text {st }}$ millennium BC, Leuven-Paris-Walpole 2014, pp. 59-84.

2014b The ceramic repertoire of the Iron Age: M.E. AuBET - F.J. NúÑEZ - L. TRELLISÓ (eds.), The Phoenician Cemetery of Tyre-Al Bass II. Archaeological Seasons 2002-2005 (Bulletin d'Archéologie et d'Architecture Libanaise. Hors-Série 9), vol. I, Beyrouth 2014 pp. 261-372.

2018 La cerámica fenicia y su función en un contexto funerario: M. GuIRGUIs (ed.), From the Mediterranean to the Atlantic: People, Goods and Ideas between East and West, $8^{\text {th }}$ International Congress of Phoenician and Punic Studies, Italy-Sardinia, CarboniaSant'Antioco 21 ${ }^{\text {th }}-26^{\text {th }}$ October 2013 (Folia Phoenicia 2), Pisa - Roma 2018, pp. 11-19.

OATES, J. - OATES, D.

$2004 \quad$ Nimrud. An Assyrian Imperial City Revealed, Norfolk 2004.

OATES, D. - REID, J.H.

1956 The Burnt Palace and the Nabu Temple; Nimrud Excavations, 1955: Iraq 18 (1956), pp. 22-38.

PEDDE, F.

2012 The Assyrian Heartland: D.T. PotTs (ed.), A Companion to the Archaeology of the Ancient Near East, New York 2012, pp. 851-866.

PICARD, C.

1995 Les oenochoés de bronze au décor égyptisant: M.H. FANTAR - M. GHAKI (eds.), Actes du III Congrès International des Études Phéniciennes et Puniques, Tunis 11-16 novembre 1991, vol. II, Tunis 1995, pp. 322-325.

PRAuSNITZ, M.W.

1982 Anhang: zum typus der kanne mit kleeblatt-mündung der roten ware in Akhziv und Samaria vom 10. bis zum 7. jahrhundert v. Chr. und zur Phönizischen expansion in den Westlichen Mittelmeerraum: H.G. NIEMEYER (ed.), Phönizier im Western (Madrider Beiträge 8), Mainz 1982, pp. 40-44.

READE, J.

2008 Nineteenth-century Nimrud: Motivation, Orientation, Conservations: J.E. CuRTIS - H McCall - D. Collon - L. AL-Gailani WerR (eds.), New Light on Nimrud. Proceedings of the Nimrud Conference $11^{\text {th }}-13^{\text {th }}$ March 2002, London (2008), pp. 1-21.

2011 The evolution of Assyrian imperial architecture: political implications and uncertainties: Mesopotamia 46 (2011), pp. 109-125.

REDISSI, T.

2007 La plaquette en ivoire de Carthage à représentation cultuelle: Africa 21 (2007), pp. 43-58. Reiche, I. - Müller, K. - Albéric, M. - Scharf, O. - WAHNing, A. - BJEOUMiKhov, A. - RadtKe, M. - SIMON, R

2013 Discovering vanished paints and naturally formed gold nanoparticles on 2800 years old Phoenician ivories using SR-FF-microXRF with the Color X-ray Camera: Analytical Chemistry 85 (2013), pp. 5857-5866. 
SANNA, A. - Lo SCHIAVO, F.

1992 Siligo (Sassari). Località Monte S. Antonio. Campagne di scavo 1990 e 1991. Relazione preliminare: lo scavo e i monumenti. I materiali rinvenuti: Bollettino di Archeologia 1315 (1992), pp. 197-203.

SECCI, R

2011 Da Nimrud a Cartagine. Rilettura iconografica di un rasoio punico al Museo del Bardo: Byrsa 19-20 (2011), pp. 129-152.

STAMPOLIDIS, N.C.

2001 Introduzione $11^{\circ}-6^{\circ}$ sec. a.C.: N.C. STAMPOLIDIS - A. KARETSOU (eds.), Il Mediterraneo orientale. Cipro - Dodecaneso - Creta $16^{\circ}-6^{\circ}$ sec. a.C. (Roma, Musei Capitolini - Palazzo Caffarelli, gennaio-aprile 2001), Atene - Roma 2001, pp. 44-103.

TALONI, M.

2012 Le oinochoai cosiddette fenicio-cipriote: origine, rielaborazione e trasformazione di una forma vascolare: AA.VV., Mode e Modelli. Fortuna e insuccesso nella circolazione di cose e idee (Officina Etruscologia 7), Roma 2012, pp. 77-98.

2013 The palmette attachment on "Phoenician" metal jugs: Annuario della Scuola Archeologica di Atene e delle Missioni Italiane in Oriente XCI, s. 3, 13 (2013), pp. 309334.

2015 Phoenician Metal Jugs: J. JimÉNEZ Ávila (ed.), Phoenician Bronzes in Mediterranean (Bibliotheca Archaeologica Hispana 45), Madrid 2015, pp. 119-146.

WINTER, I.J.

1976a Phoenician and North Syrian Ivory Carving in Historical Context: Questions of Style and Distribution: Iraq 38, 1 (1976), pp. 1-22.

1976b Carved Ivory Furniture Panels from Nimrud: A Coherent Subgroup of the North Syrian Style: Metropolitan Museum Journal 11 (1976), pp. 25-54. 


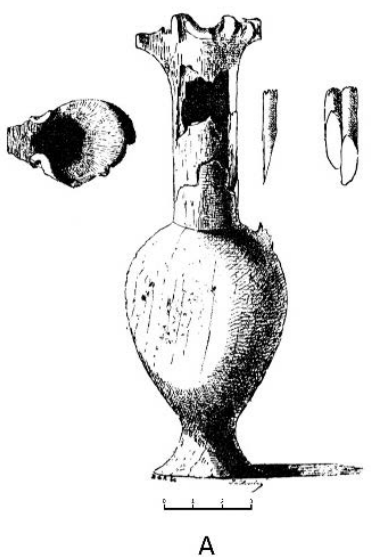

A

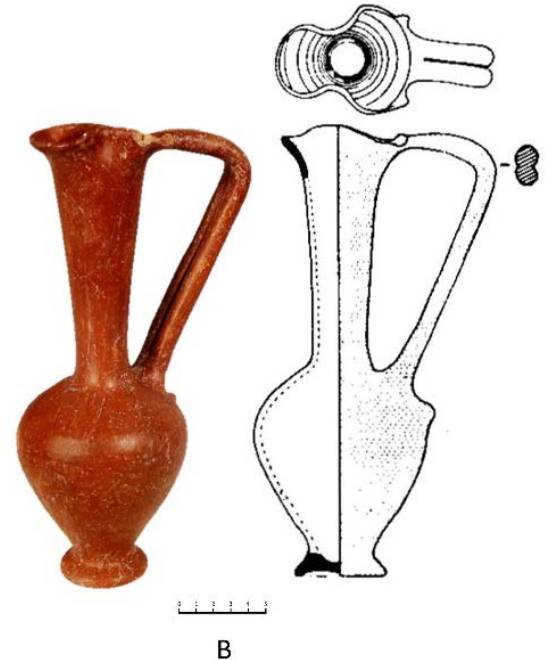

B

Fig. 1 - Riproduzione grafica della brocca eburnea dalla necropoli cartaginese di Douïmès (A; Culican 1968, 281, fig. 2); brocca in Red-Slip proveniente dalla necropoli cipriota di Amatunte, Tomba 384 (B; Stampolidis 2001, 88, fig. 192; Bikai 1987a, tav. XIV, 355); elaborazione dell'Autore.

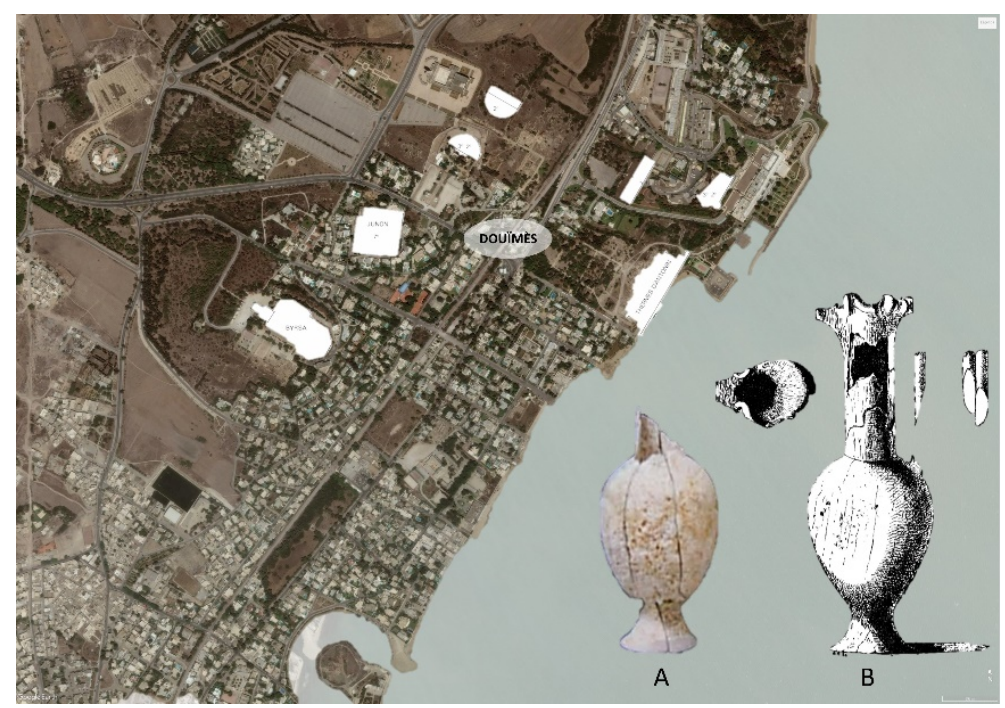

Fig. 2 - Localizzazione del settore necropolare di Douïmès a Cartagine (da: Google Earth; Benichou-Safar 1982, fig. 139); fotografia (A; Khelifi 2017, 34, n. 52) e disegno (B; Culican 1968, 281, fig. 2) della brocchetta in avorio rinvenuta nel 1896; rielaborazione dell'Autore. 


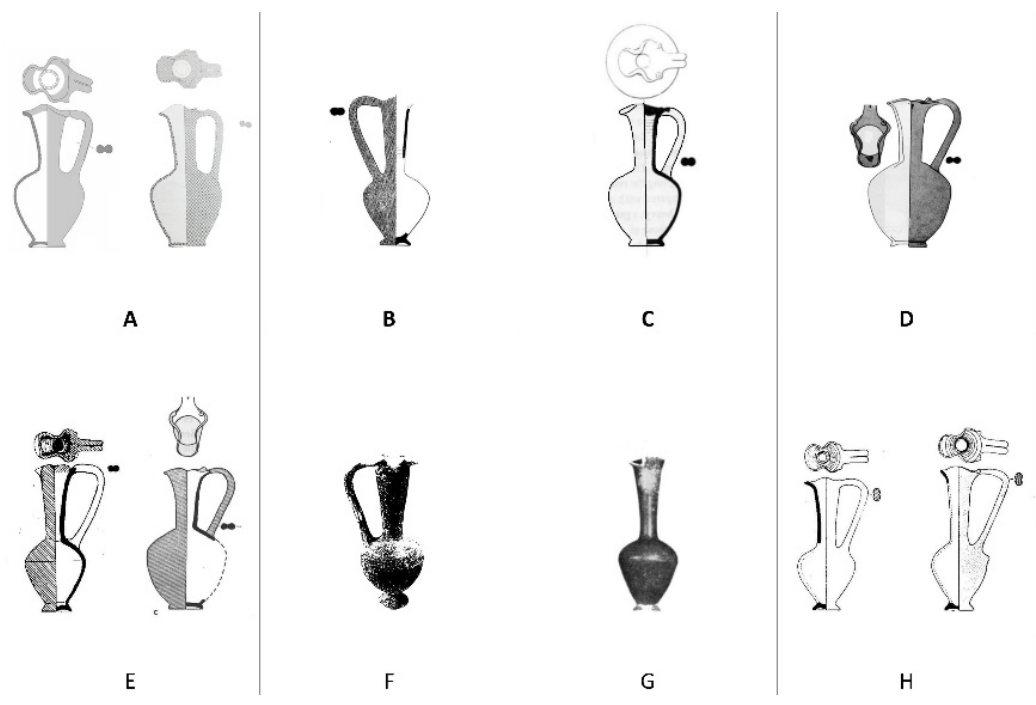

Fig. 3 - Forme ceramiche in Red-Slip del IX sec. a.C. dall'area levantina e da Cipro: Tiro (A; Núñez 2004, fig. 173; Núñez 2018, fig. 5a5), Joya (B; Vibert Chapman 1972, fig. 27:261), Khaldé (C; Culican 1982, fig. 5:d), Khirbet Silm (D; Culican 1982, fig. 8:a), Akhziv (E; Mazar 2001, fig. 9:192; Prausnitz 1982, fig. 3), Tell es-Safi (F; Amiran 1969, fig. 281), Kourion (G; Culican 1982, tav. 5:e), Amathus (H; Bikai 1987a, tav. XIV:353, 355); rielaborazione dell'Autore (non in scala).

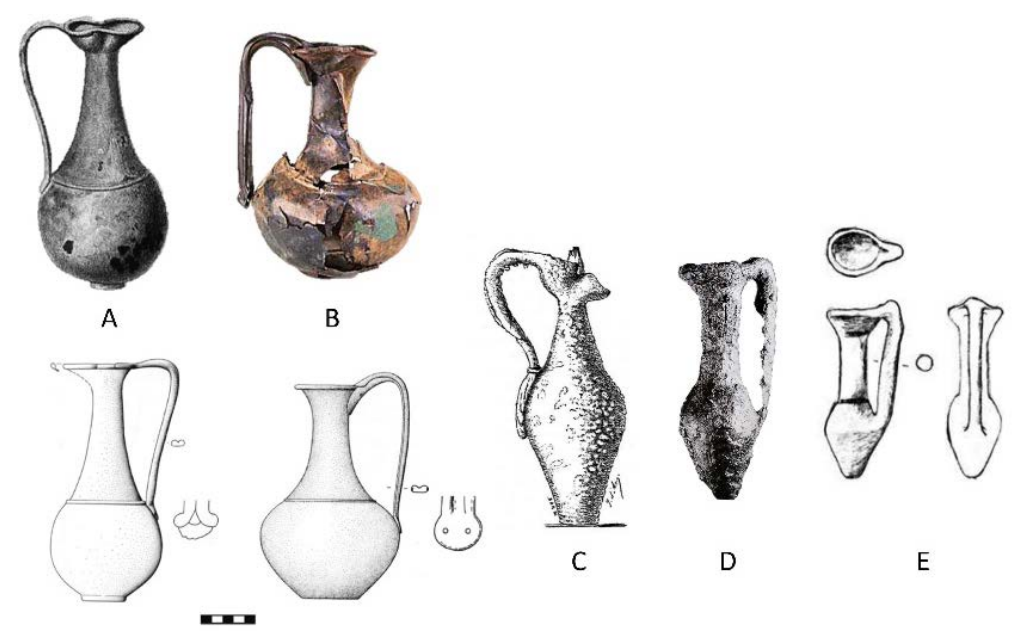

Fig. 4 - Brocche in metallo da Cipro nella Collezione Cesnola (A; Taloni 2015, figg. 1:1, 7), dall'Antro Ideo a Creta (B; Kourou 2012, fig. 1a), dalla necropoli di Cartagine (C; Delattre 1897, fig. 88); fotografia e riproduzione grafica della brocchetta miniaturistica dalla Sardegna (D-E; Lo Schiavo 2000, fig. 6:4 a-b; Ialongo 2010, vol. II, fig. 24); rielaborazione dell'Autore. 


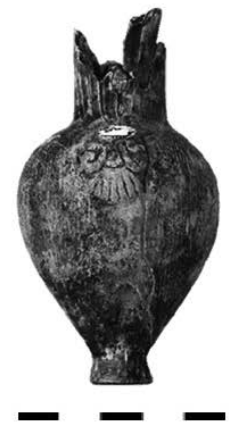

A

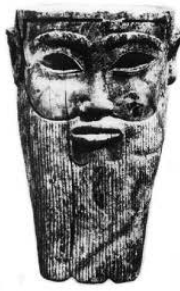

B

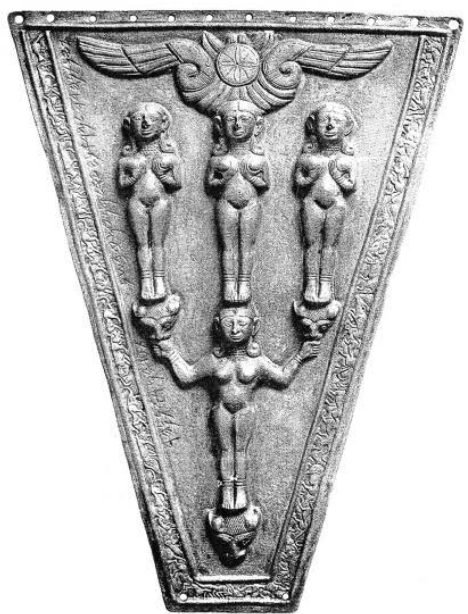

C

Fig. 5 - Brocchetta (A; Taloni 2015, fig. 11:3), testina eburnea (B; Jiménez Ávila 2015, fig. 8:2) e frontalino in bronzo con iscrizione aramaica (C; Bonatz 2014, tav. XXVI) dall'Heraion di Samo; rielaborazione dell'Autore.
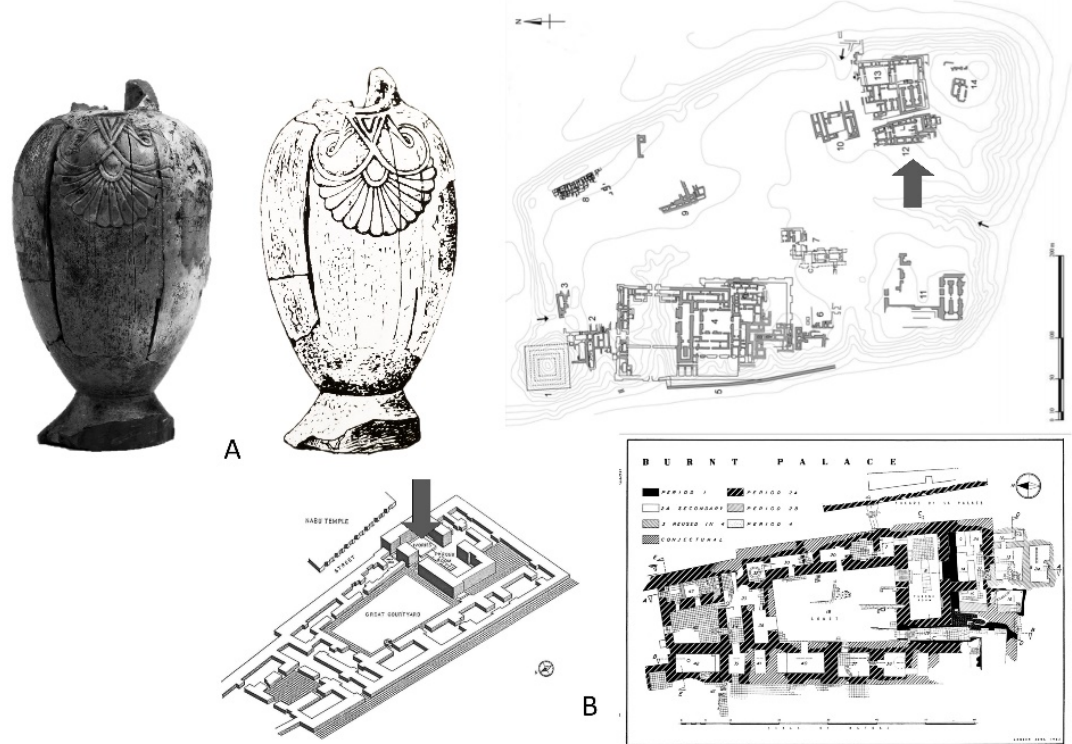

Fig. 6 - Riproduzione fotografica e grafica della brocchetta eburnea (A; Barnett 1935, tav. XXIV:1) proveniente dal Burnt Palace nella cittadella di Nimrud (B; Barnett 1957; Mallowan 1954, tav. XI; Oates - Oates 2004, fig. 78; Gabellone - Scardozzi 2007, fig. 30); rielaborazione dell'Autore. 

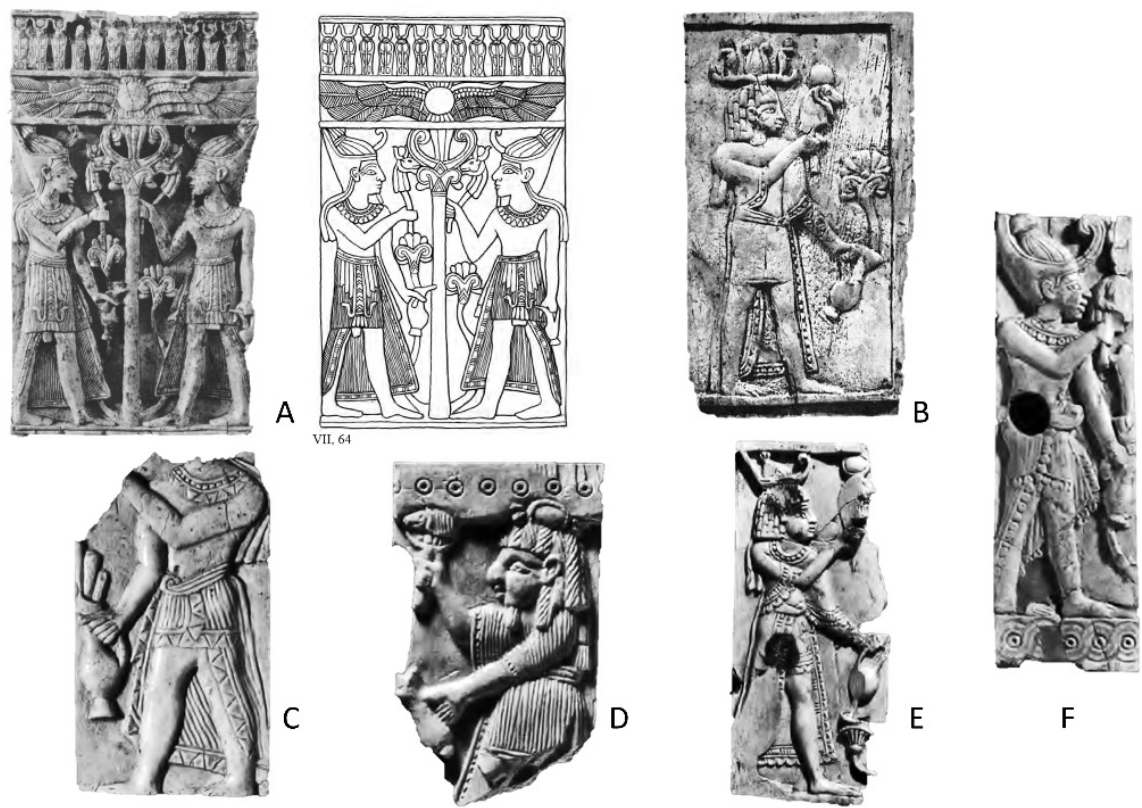

$\mathrm{F}$

Fig. 7 - Pannelli eburnei dal Forte Salmanassar a Nimrud: dalla Room SW.12 (A; Herrmann - Laidlaw 2013b, 57, fig. 3a; Winter 1976b, fig. 15), da SW.7 (B; Mallowan Herrmann 1974, tav. XCV:87) e da SW. 37 (C; Herrmann 1986, tav. 79:347; D; tav. 81:357; E; tav. 76:337; F; tav. 80:350); rielaborazione dell’ Autore.

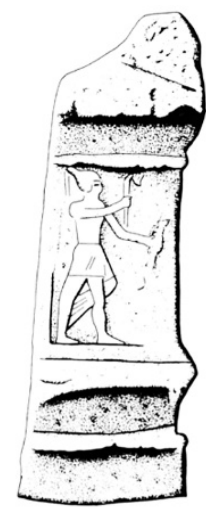

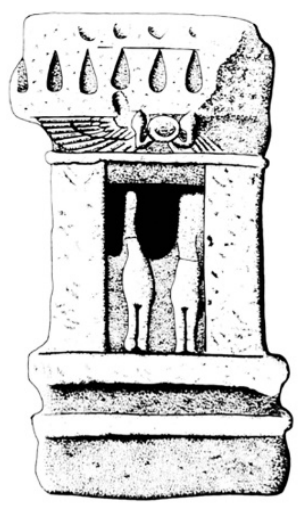

A

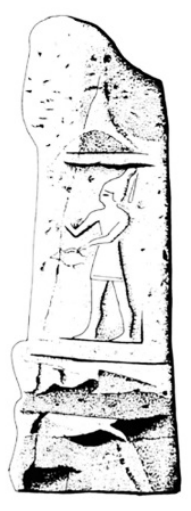

Fig. 8 - Monumento lapideo (naiskos) da Sidone (A; Gubel 2016, fig. 9) e rasoio in bronzo da Cartagine (B; Cecchini 2005, fig. 8); rielaborazione dell’Autore. 


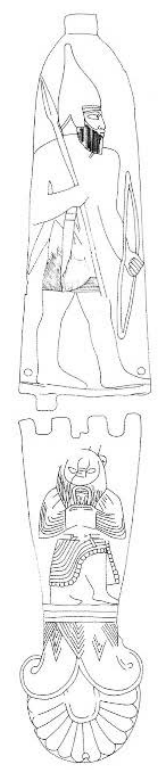

A

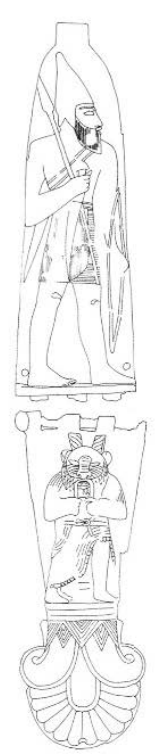

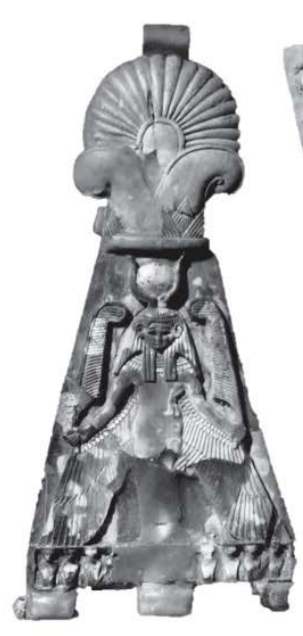

B
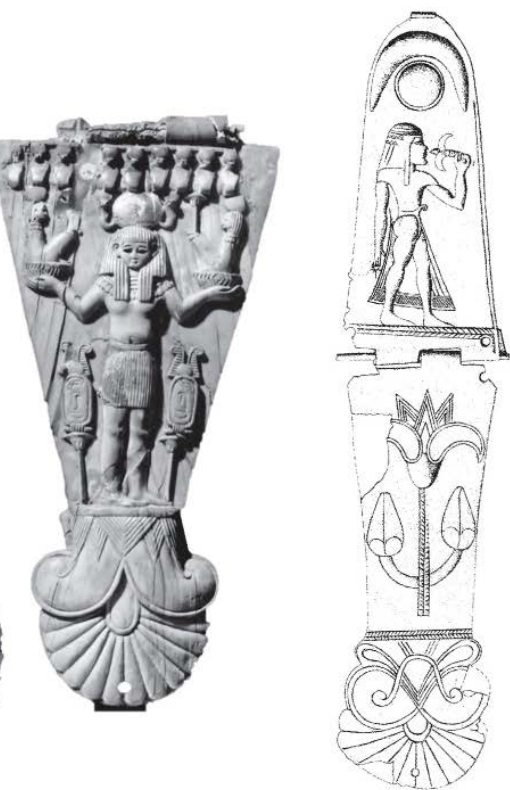

C

Fig. 9 - Frontalini in avorio da Nimrud (A; Herrmann - Laidlaw - Coffey 2009, tav. 78:254; B; Gubel 2005, fig. 13) e in bronzo da Enkomi (C; Gubel 2005, fig. 20); rielaborazione dell'Autore. 